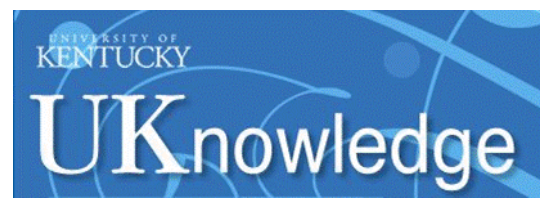

University of Kentucky

UKnowledge

$10-1-2010$

\title{
Alterations in Multiple Measures of White Matter Integrity in Normal Women at High Risk for Alzheimer's Disease
}

\author{
Brian T. Gold \\ University of Kentucky, brian.gold@uky.edu \\ David K. Powell \\ University of Kentucky, dkpowe2@uky.edu \\ Anders $\mathrm{H}$. Andersen \\ University of Kentucky, anders@mri.uky.edu \\ Charles D. Smith \\ University of Kentucky, csmith@mri.uky.edu
}

Follow this and additional works at: https://uknowledge.uky.edu/neurobio_facpub

Part of the Bioimaging and Biomedical Optics Commons, and the Neuroscience and Neurobiology Commons

Right click to open a feedback form in a new tab to let us know how this document benefits you.

\section{Repository Citation}

Gold, Brian T.; Powell, David K.; Andersen, Anders H.; and Smith, Charles D., "Alterations in Multiple Measures of White Matter Integrity in Normal Women at High Risk for Alzheimer's Disease" (2010). Neuroscience Faculty Publications. 9.

https://uknowledge.uky.edu/neurobio_facpub/9

This Article is brought to you for free and open access by the Neuroscience at UKnowledge. It has been accepted for inclusion in Neuroscience Faculty Publications by an authorized administrator of UKnowledge. For more information, please contact UKnowledge@lsv.uky.edu. 


\section{Alterations in Multiple Measures of White Matter Integrity in Normal Women at High Risk for Alzheimer's Disease}

\section{Digital Object Identifier (DOI)}

http://dx.doi.org/10.1016/j.neuroimage.2010.05.036

\section{Notes/Citation Information}

Published in Neurolmage, v. 52, issue 4, p. 1487-1494.

(c) 2010 Elsevier Inc. All rights reserved.

This manuscript version is made available under the CC-BY-NC-ND 4.0 license http://creativecommons.org/licenses/by-nc-nd/4.0/ 
(C) 2010 Elsevier Inc. All rights reserved.

This manuscript version is made available under the CC-BY-NC-ND 4.0 license

http://creativecommons.org/licenses/by-nc-

nd/4.0/ 


\title{
Alterations in multiple measures of white matter integrity in normal women at high risk for Alzheimer's disease
}

\author{
Brian T. Gold ${ }^{a, b},{ }^{*}$, David A. Powell ${ }^{b}$, Anders H. Andersen ${ }^{a, b}$, and Charles D Smith ${ }^{a, b, c}$ \\ aDepartment of Anatomy and Neurobiology, University of Kentucky, Lexington, KY 40536 \\ bMagnetic Resonance Imaging and Spectroscopy Center, University of Kentucky, Lexington, KY \\ 40536 \\ 'Department of Neurology, University of Kentucky, Lexington, KY 40536
}

\begin{abstract}
There is evidence that disruption of white matter (WM) microstructure is an early event in the course of Alzheimer's disease (AD). However, the neurobiological bases of WM microstructural declines in presymptomatic $\mathrm{AD}$ are unknown. In the present study we address this issue using a multimodal imaging approach to the study of presymptomatic AD. Participants were 37 high risk (both family history of dementia and one or more APOE4 alleles) women and 20 low risk (neither family history nor APOE4) women. Groups were matched for age, education, neuropsychological performance, and vascular factors that could affect white matter. Whole-brain analyses of diffusion tensor imaging data [including fractional anisotropy (FA), mean diffusivity (MD), axial diffusivity (DA) and radial diffusivity (DR)] and volumetric comparisons of medial temporal lobe (MTL) structures were conducted. Results indicated equivalent entorhinal cortex and hippocampal volumes between risk groups. Nevertheless, the high risk group showed decreased microstructural integrity in WM tracts with direct and secondary connections to the MTL. The predominant alteration in WM integrity in the high AD-risk group was decreased FA not solely driven by either DA or DR changes alone in regions where no MD changes were observed. A second pattern observed in a smaller number of regions involved decreased FA and increased DR. These results suggest that disconnection of MTL-neocortical fiber pathways represents a very early event in the course of $\mathrm{AD}$ and suggest that demyelination may represent one contributing mechanism.
\end{abstract}

\section{Keywords}

diffusion tensor; fractional anisotropy; magnetic resonance imaging; Alzheimer's disease; apolipoprotein E; myelin

\section{Introduction}

Alzheimer's disease (AD) has traditionally been viewed as a disease of grey matter - a neuronal/synaptic polioencephalopathy. However, there is pathological evidence that

\footnotetext{
(C) 2010 Elsevier Inc. All rights reserved.

"Corresponding Author: Dr. Brian T. Gold, MN 214, University of Kentucky Medical Center, 800 Rose Street, Lexington, KY 40536; brian.gold@uky.edu.

Publisher's Disclaimer: This is a PDF file of an unedited manuscript that has been accepted for publication. As a service to our customers we are providing this early version of the manuscript. The manuscript will undergo copyediting, typesetting, and review of the resulting proof before it is published in its final citable form. Please note that during the production process errors may be discovered which could affect the content, and all legal disclaimers that apply to the journal pertain.
} 
disruption of white matter (WM) microstructure is an early event in the course of AD (Brun and Englund, 1986; Englund et al., 1988). Concordant with this pathology data, evidence of WM disruption in $\mathrm{AD}$ has been demonstrated by non-invasive diffusion tensor imaging (DTI) (reviewed in Stebbins and Murphy, 2009). DTI is sensitized to the random motion of water molecules as they interact within tissues, thus reflecting characteristics of their immediate structural surroundings. In WM, the motion of water molecules is hindered more in directions orthogonal to the main fiber direction than along the fiber, and thus diffusion tends to be anisotropic. Diffusion anisotropy can be measured by DTI by means of fractional anisotropy, an index of overall tissue microstructural integrity (Pierpaoli and Basser, 1996). A body of data from DTI studies has demonstrated that reduced WM integrity is not only evident in the frontal, temporal and parietal lobes of individuals with $\mathrm{AD}$, but also in its typical early prodromal state of mild cognitive impairment (MCI) (reviewed in Stebbins and Murphy, 2009).

These findings have motivated the question of whether WM microstructural changes are present in cognitively normal individuals at high risk for future $\mathrm{AD}$, a potential target group for emerging interventions. Several recent studies have demonstrated that individuals at high risk for future AD based on an apolipoprotein-E4 (APOE4) allele show reduced fractional anisotropy (FA) in certain brain regions compared to low risk groups (Nierenberg et al., 2005; Persson et al., 2006; Smith et al., 2008). These studies used manual region-of-interest (ROI) tracing (Nierenberg et al., 2005), voxel-based morphometry (Persson et al., 2006) or tract-based spatial statistics implemented in FSL software (Smith et al., 2008). In general, the decreases in WM integrity reported in these studies of normal subjects were of smaller magnitude and more confined to tracts associated with medial temporal lobe structures than observed in studies of AD subjects. For example, presymptomatic AD studies have reported reduced FA in the cingulum (Persson et al., 2006; Smith et al., 2008), parahippocampal WM (Nierenberg et al., 2005; Persson et al., 2006; Smith et al., 2008) and inferior longitudinal fasciculus (Smith et al., 2008). These data suggest that changes in WM integrity precede cognitive decline in $\mathrm{AD}$, which may prove important for early $\mathrm{AD}$ detection by non-invasive imaging.

However, there currently exist at least two knowledge gaps concerning WM microstructural declines in presymptomatic $\mathrm{AD}$, resulting from the fact that previous DTI studies of WM integrity in presymptomatic $\mathrm{AD}$ have focused almost exclusively on the FA metric. One resulting knowledge gap concerns whether WM microstructural changes in presymptomatic $\mathrm{AD}$ are independent of or secondary to classically described grey matter medial temporal lobe (MTL) degeneration. Neuropathology affects the hippocampus and entorhinal cortex (ERC) early in the course of AD (Braak and Braak, 1991; Hyman et al., 1984), and atrophy of these MTL structures is evident with MR imaging early in the AD disease process (Convit et al., 1997; de Leon et al., 2004; Jack et al., 1997). In addition, recent results suggest that hippocampal and ERC volumes can be reduced in presymptomatic $\mathrm{AD}$, several years prior to MCI diagnosis (Martin et al., 2008; Smith et al., 2007). It is thus important for studies of WM integrity to assess MTL volume and to determine its potential contribution to reduced integrity of MTL WM tracts in presymptomatic AD.

A second knowledge gap concerns the pattern of diffusivity characteristics beyond FA that characterize presymptomatic AD. The FA metric represents a weighted average of distinct components of the diffusion tensor and provides an overall assessment of WM integrity (Pierpaoli and Basser, 1996). However, a comprehensive understanding of WM microstructural declines requires joint consideration of FA, mean diffusivity, radial diffusivity, and axial diffusivity (Assaf and Pasternak, 2008; Burzynska et al., 2009). Mean diffusivity (MD) is an average of all three eigenvalues of the diffusion tensor and depends on the density of physical obstructions such as membranes and the distribution of water 
molecules between different cell compartments (Beaulieu, 2002; Sen and Basser, 2005). The diffusion tensor can also be decomposed into three mutually orthogonal eigenvectors. In particular, the tensor can be separated to reflect the diffusion coefficient along the direction of maximal 'apparent' diffusion ( $\lambda_{1}$ or 'axial diffusivity'), and the diffusion coefficients along two orthogonal directions embedded in the plane perpendicular to the main diffusion direction $\left(\lambda_{2}\right.$ and $\left.\lambda_{3}\right)$. The second and third eigenvalues can be averaged and expressed as 'radial diffusivity'. These component (axial and radial) diffusivities have been linked with different underlying pathologies (Song et al., 2002; Sen and Basser, 2005).

One way to begin to characterize the neurobiological bases of recently described FA decreases in presymptomatic $\mathrm{AD}$ would be to identify group differences in MD, DR, and DA within regions showing FA decreases. This approach allows for a more detailed understanding about WM integrity decline than separate consideration of FA, MD, DR and DA (Assaf and Pasternak, 2008; Burzynska et al., 2009). For example, a finding of increased MD in regions showing decreased FA would suggest reductions in microstructural integrity that are in part associated with macroscopic tissue loss and increased brain water content (Sen and Basser, 2005). In contrast, decreased FA without increased MD suggests microstructural changes to WM fibers that are not due to gross tissue loss (Sen and Basser, 2005). A reduction in FA along with increased DR has been linked with loss of myelin in multiple sclerosis (Ciccarelli et al., 2006) and in animal studies of experimentally induced myelin loss (Song et al., 2002; Song et al., 2005; Sun et al., 2006). Finally, a decrease in FA and DA without a DR increase has been reported in both rodents and humans with axonal damage associated with axonal swelling, fragmentation and organelle accumulations (Concha et al., 2006; Sun et al., 2006).

In the present exploratory study we address these two knowledge gaps concerning WM integrity reductions in presymptomatic AD using a multimodal imaging approach. We first assessed the macrostructural integrity (i.e., volume) of the two MTL regions which have been classically linked with AD pathology and MRI-based brain atrophy: the ERC and hippocampus. Next we assessed the pattern of WM microstructural changes associated with being at high risk for $\mathrm{AD}$. In particular, we sought to provide information about potential neurobiological bases of decreased WM decline in presymptomatic AD through assessment of the combined pattern of whole-brain changes in FA, MD, DA and DR. Finally, we assessed the relationship between these WM metrics and hippocampal volume.

\section{Methods}

\section{Participants}

Participants were 37 high risk (both family history of dementia and one or more APOE4 alleles) women and 20 low risk (neither family history nor APOE4) women who ranged in age from 50 to 70 years old (Table 1). All participants in the present study are involved in a larger ongoing University of Kentucky longitudinal imaging study of normal persons who vary in their risk of AD based on family history of dementia and APOE allele status (Smith et al., 2008). Only right-handed females are recruited for the larger longitudinal imaging study, from which the present subjects were drawn, because the comprehensive imaging protocol includes functional magnetic resonance imaging of language-based tasks, a cognitive domain in which gender and handedness can influence activation. Participants in the present study were selected from the larger cohort on the basis of (1) having an available DT image; (2) age (in order to match groups for mean age) and (3) the absence of any detectable vascular pathology (small or large vessel infarcts, microhemorrhages, or significant periventricular hyperintensities). The FA data of some of the present subjects have been reported in a previous study (Smith et al., 2008). 
Inclusion criteria other than sex and handedness in the imaging protocol include generally good health without memory complaints, stable medication regimen, being between the age of 40 and 90 years, vision correctable to at least 20/50, and knowledge of family history. Family history is considered positive if one or more first degree relatives had late-onset dementia. Exclusions are stroke or other cerebral injury, psychoactive medications, except stable doses of serotonin reuptake inhibitors, active depression, significant head injury (operationally defined as a loss of consciousness greater than five minutes), significant psychiatric history, claustrophobia, metallic implants in the head or neck, pacemakers, major surgery within three months, or death of one or both parents before age 65 .

Participants from either risk group who are selected for scanning undergo standardized psychometric testing which includes the Mini Mental State Examination (MMSE), Trails A and B, FAS Letter Fluency (LF), Wechsler Adult Intelligence Scale (WAIS ) Block Design (Block Des) and Vocabulary (Vocab), 15-item Boston Naming Test (BNT), Wechsler Memory Scale (WMS) Logical Memory I (LM Imm) and II (LM Del), Visual Reproduction immediate and delayed (VR Imm and VR Del), Digit Span (DS), Spatial Span (SS), Selective Reminding Test Immediate and Delayed (SRT Imm \& SRT Del), and the Beck depression inventory (Beck). Abbreviations are given for reference to Table 1 . These tests are given to exclude persons with cognitive impairment and to document equivalent normal cognitive performance between risk groups. All subjects had an APOE allele determination, using polymerase chain reaction (PCR) amplification followed by enzymatic digestion and separation of DNA fragments by agarose gel electrophoresis. Informed consent was obtained from each enrollee under an approved University of Kentucky Medical Institutional Review Board protocol.

\section{MRI Acquisition Procedures}

Data were collected on a 3 Tesla Siemens Trio scanner at the University of Kentucky. An 8channel imaging coil was used. Comfortable, disposable foam earplugs were used to dampen scanner noise. Comfortable foam padding was used to limit head motion within the coil and to further dampen scanner noise.

Diffusion Tensor Imaging (DTI) Acquisition-Whole-brain (40 slices) diffusion tensor images were acquired with 3 averages in each of 12 encoding directions $\left(\mathrm{B}_{0}\right.$ images were averaged 6 times; TR $=14600 \mathrm{~ms}$, TE= $96 \mathrm{~ms}$; resolution $1.8 \times 1.8 \times 3.0 \mathrm{~mm}$ ) using a fluid attenuated inversion recovery (FLAIR) double-refocused spin echo sequence.

Structural MRI-Participants underwent structural MRI during the same scanning session as diffusion-weighted imaging. A three-dimentional magnetization-prepared rapid gradient echo (MP-RAGE) sequence was used with parameters: repetition time (TR) $=2100 \mathrm{~ms}$, echo time $(\mathrm{TE})=2.93 \mathrm{~ms}$, inversion time $(\mathrm{TI})=1100 \mathrm{~ms}$, flip angle $=12^{\circ}$, field of view (F.O.V.) $=224 \times 256 \times 192 \mathrm{~mm}$, sagittal partitions, yielding isotropic $1 \mathrm{~mm}^{3}$ voxels.

\section{MRI Analysis Procedures}

\section{Hippocampal and ERC volumes-FreeSurfer software}

(http://surfer.nmr.mgh.harvard.edu) was used for cortical parcellation (Desikan et al., 2006; Fischl et al., 2004b) and subcortical segmentation (Fischl et al., 2002; Fischl et al., 2004a). The standard data processing stream in FreeSurfer involves intensity normalization for removal of the B1 bias field, skull stripping, and Markov random field modeling for segmentation and gray matter volume labeling, incorporating atlas-based anatomical class priors. Individual structure volumes are in turn computed from labeled voxels and normalized to total intracranial volume (ICV). 
DTI preprocessing-T2 Images were inspected for visible WM hyperintensities by an experienced neurologist (Dr. Charles Smith). Diffusion volumes were motion corrected and averaged using the Linear Image Registration Tool in FSL software (FLIRT:

http://www.fmrib.ox.ac.uk/fsl). Corrections for motion and residual eddy current distortion were performed using a 12-parameter affine transformation of the images from each direction to the minimally eddy current distorted T2-weighted DTI volume that has no diffusion weighting.

Fractional anisotropy and diffusivity map calculations-Each participant's voxelwise diffusion tensor was calculated using nonlinear estimation (Basser and Pierpaoli, 1996). Maps for FA, mean diffusivity (MD), axial diffusivity (DA) and radial diffusivity (DR) were extracted using procedures described elsewhere (Basser et al., 1994). Briefly, for FA maps, three eigenvectors that define the diffusion ellipsoid were calculated in each voxel from the diffusion tensor. These eigenvectors correspond to three eigenvalues, which represent the magnitude of diffusivity in the three principal directions relative to the maximum in each voxel. Thus the diffusion tensor is based on directions and magnitudes of diffusivity within the brain, independent of scanner coordinates. Based upon the three principal diffusivities, FA is calculated in each voxel (Basser and Pierpaoli, 1996). Mean diffusivity is a scalar measure of the total amount of diffusion within a voxel and was calculated as described in Basser and Pierpaoli (1996). Measures of axial $\left(\lambda_{1}\right)$ and radial $\left[\left(\lambda_{2}\right.\right.$ $\left.+\lambda_{3}\right) / 2$ ] diffusivity were computed as described in Song et al. (2002).

\section{Nonlinear Registration of FA and Diffusivity Maps into Standard Space-}

Registration of FA images into MNI152 space and subsequent voxelwise analyses followed a series of procedures known as Tract-Based Spatial Statistics [TBSS v1.2; (Smith et al., 2006)], part of the publicly available image processing software FSL 4.0 (http://www.fmrib.ox.ac.uk/fsl/tbss/). TBSS was used because its algorithms for alignment of FA images from multiple subjects into a common space have been extensively tested and validated [Smith et al., 2006]. In addition, the technique does not require spatial smoothing of images, which can produce different results depending upon the kernel size (Jones et al., 2005). All subjects' FA images were first aligned to a common registration target (the image to which the least amount of warping was required for all other subjects to align to it) using a nonlinear registration approach based on free-form deformations and B-Splines (Rueckert et al., 1999). FA datasets were then affine registered and resampled to $1 \times 1 \times 1 \mathrm{~mm}$ MNI152 space. The exact transformations derived from the FA maps were then applied to the other diffusivity maps (MD/DR/DA) for matched processing of all image volumes. All subsequent processing was carried out in this standardized space.

The MNI-transformed FA images from all subjects were averaged to create a mean FA image. The mean FA image was then entered into the tract skeleton generator, which calculates a tract representation 'common' to all participants. The tract skeleton was then thresholded at an FA value of 0.2 in order to exclude regions that likely comprise multiple tissue types or fiber orientations. Each participant's aligned FA image was subsequently projected onto the FA skeleton in order to account for residual misalignments between participants after the initial nonlinear registration. This effectively aligns each participant's FA image with the skeleton and is achieved for each skeleton voxel by searching perpendicular to the local skeleton structure for the maximum value in the subject's FA image. The result is a common tract skeleton with each subject's FA image aligned to this common skeleton. The skeleton is thus filled with each participant's FA values from the centers of the nearest relevant tracts. Thus, the data are in the form of a sparse $4 \mathrm{D}$ image, with the fourth dimension being subject ID. Each subject's MD, DA and DR maps in MNI space were then projected onto the common tract skeleton, using the pipeline for non-FA 
data provided by TBSS, which employs the projection vectors from each individual's FA-toskeleton transformation (Smith et al., 2006).

A statistical threshold of $\mathrm{P}<0.001$ (uncorrected) was employed for separate between-group comparisons of FA, MD and component diffusivity (DR and DA). The main purpose of these analyses was to characterize the potential neurobiological bases of FA changes previously reported in presymptomatic AD. Group difference maps of MD, DR and DA were thus masked by regions showing decreased FA in the high risk group (there were no regions of decreased FA in the low risk group). Restricting between-group analyses of MD/ DR/DA maps to voxels showing decreased FA in the high risk group also served to reduce the type I error risk by reducing the number of comparisons performed. Age was included as a covariate in all WM integrity analyses (FA/MD/DR/DA). All statistical maps were dilated from the TBSS skeleton for visualization purposes.

\section{Results}

Demographic and psychometric scores were compared between risk groups (Table 1). The high and low AD-risk groups of women did not differ in mean age or education. Because no correction was made for the 14 multiple comparisons associated with the psychometric test scores in Table 1, we chose to conservatively interpret a P-value between 0.01 and 0.05 as indicating marginal significance, and a P-value less than 0.01 as significant. None of the 14 psychometric tests met our threshold P-value of $<0.01$ for risk group difference. The high AD-risk group scored lower on the letter fluency test and this difference was marginally significant $(\mathrm{P}=0.03)$.

To test for potential differences between risk groups related to vascular factors or estrogen replacement that might affect white matter, Chi-Square analysis using Pearson's criterion without correction for multiple comparisons was performed on the following categorical variables (Present/Not Present): current smoking, past smoking, current estrogen replacement, past estrogen replacement, hypercholesterolemia, hypertension and diabetes. There were three subjects with stable diabetes in the study, one in the low and two in the high AD-risk group. None of the $\mathrm{P}$-values in these comparisons was less than $\mathrm{P}=0.40$, demonstrating that the frequencies of these factors were the same between risk groups.

\section{Hippocampus and Entorhinal Cortex Volume}

Figure 1 displays the mean hippocampal and entorhinal cortex volumes in normal high and low AD-risk groups. Separate ANCOVAs were conducted with MTL volume (hippocampal or ERC) as the dependent variable, risk group as the independent variable of interest and age and ICV as covariates of no interest. Because there were no hemisphere by risk group interactions in either MTL ROI volume (both F's < 1.0), the average (left-right) hippocampal and ERC volumes were entered into between-group comparisons. The high and low AD-risk groups did not differ in hippocampus volume, $\mathrm{F}(1,53)=0.12$ for $\mathrm{P}=0.73$, or in $\mathrm{ERC}$ volume $\mathrm{F}(1,53)=0.42$ for $\mathrm{P}=0.52$.

\section{White Matter Microstructural Integrity}

Figure 2 presents the results from the group comparisons of FA and MD. The anatomic underlay is the MNI-space registered target FA image. Maps are referenced to a standard human white matter atlas (Mori et al., 2005). The high AD-risk group showed decreased FA (shown in red) compared to the low risk group in a number of regions, prominently including the fornix and inferior longitudinal fasciculus (ILF) in the temporal lobe and anterior portions of the inferior fronto-occipital fasciculus (IFOF) / uncinate fasciculus (UNC) in the frontal lobe. There were no regions in which the low AD-risk group showed 
decreased FA compared to the high risk group. Within regions of decreased FA, there were only two regions of increased MD in the high risk group (shown in orange): the genu and the right IFOF/ILF.

Figure 3 presents the results from the comparison of component diffusivities [radial diffusivity (DR) and axial diffusivity (DA)] within regions showing group-based FA differences. Within regions of decreased FA in the high AD-risk group, the majority of component diffusivity changes were characterized by increased DR. Regions showing increased DR in the high risk group (shown in blue) were bilateral portions of the ILF in the temporal lobe and bilateral portions of the IFOF / UNC in the frontal lobe. In contrast, there were only a few small regions in which the high risk group showed altered DA compared to the low risk groups. The high risk group showed decreased DA compared to the low risk group (shown in yellow) in a rostral portion of the cingulum. Finally, the high risk group showed decreased DA in only a few scattered voxels (not shown).

One of the regions showing decreased WM integrity in the high risk group, the fornix, represents the principal efferent pathway of the hippocampus. Despite the equivalence of hippocampal volume between groups, within-group variability in hippocampal volume could affect the size of WM microstructural effects in the fornix or other MTL tracts. This possibility was explored in a set of supplementary analyses which considered the effects of including normalized hippocampal volume as a covariate in the group comparisons of FA/ MD/DR/DA. Including normalized hippocampal volume as a covariate had minimal effects on the between-group WM microstructural results and did not alter the finding of reduced FA in the fornix in the high AD-risk group (Figure 4). Rather, controlling for hippocampal volume revealed an additional effect of reduced FA (and increased DR) in the high AD-risk group in the caudal portion of the left cingulum, a tract with direct connections between the MTL and posterior cingulate cortex.

\section{Discussion}

Our results demonstrate white matter (WM) microstructural integrity reductions in normal individuals at high risk for Alzheimer's disease (AD) in the absence of medial temporal lobe (MTL) atrophy. Reductions of microstructural integrity of MTL fiber tracts, in addition to other pathways, have been reported in previous DTI studies of MCI/AD (reviewed in Stebbins and Murphy, 2009). However, atrophy of the entorhinal cortex (ERC) and hippocampus is observed in MCI/AD (Convit et al., 1997; de Leon et al., 2004; Jack et al., 1997) and may have contributed to the reduced integrity of MTL tracts observed in previous studies. The present results demonstrate that WM microstructural integrity reductions in tracts with direct and secondary connections to MTL structures are not merely a consequence of classic AD-related ERC/hippocampal atrophy and may precede macroscopic degeneration in these regions.

The WM tracts showing decreased integrity in presymptomatic AD represent connections among structures involved in episodic memory function (Colchester et al., 2001). The high AD-risk group showed decreased fractional anisotropy (FA) in the fornix, cingulum, inferior longitudinal fasciculus (ILF) and rostral portions of the inferior fronto-occipital fasciculus (IFOF). The fornix is the major efferent tract of the hippocampus, connecting it with the septal frontal region, the mamillary bodies of the hypothalamus, and the anterior nuclei of the thalamus. The caudal portion of the cingulum contains fibers that connect the posterior cingulate cortex with the hippocampus and parahippocampal gyrus (Mufson and Pandya, 1984). The ILF contains connections between occipital and temporal regions, and the IFOF contains connections between occipital regions and ventrolateral prefrontal cortex (Catani et al., 2002). Both the ILF and IFOF have secondary connections to the MTL (Catani et al., 
2002). Our results using in vivo DT imaging are consistent with pathological data suggesting that limbic-neocortical pathways are preferentially affected early in AD (Braak and Braak, 1996).

Joint consideration of the major components of the diffusion tensor revealed several patterns of WM integrity changes in the presymptomatic AD group. A predominant pattern was reduced FA in the absence of mean diffusivity (MD) differences. Only a small portion of the ILF/IFOF and genu showed the combined pattern of decreased FA and increased MD. The absence of MD differences within regions of decreased FA suggests mild microstructural loss without gross tissue loss (Sen and Basser, 2005), a result which converges with our findings of equivalent hippocampal and entorhinal volumes between risk groups. The absence of MD differences in regions of decreased FA in presymptomatic AD contrasts with findings from studies of symptomatic $\mathrm{AD}$, in which FA and MD are often negatively correlated (Fellgiebel et al., 2004; Medina et al., 2006; Zhang et al., 2007). Our results suggest that FA and MD are not necessarily correlated and that FA changes can be observed in the absence of MD changes associated with gross tissue loss.

A second pattern of WM integrity changes in the presymptomatic group was revealed through joint analyses of FA and component (axial and radial) diffusivities. Results revealed that the majority of FA reductions in the high risk group were not solely driven by either axial diffusivity (DA) or radial diffusivity (DR) changes alone. Regions showing decreased FA in the high risk group not solely driven by alterations in either DA or DR alone included a caudal portion of the fornix, periventricular regions and a portion of the ILF. Reductions in FA not solely driven by either DA or DR changes alone, in regions where MD is not increased, may reflect a subtle mixture of axonal and myelin damage, possibly resulting from minor loss of fibers and their surrounding myelin sheath (Sen and Basser, 2005; Burzynska et al., 2009). In addition to such potential microstructural changes, the pattern of reduced FA not solely driven by either DA or DR changes alone may also reflect voxel-level macrostructural variables such as decreased coherence in the orientation of axons (Bennett et al., 2010).

In WM tracts where changes in component diffusivities were noted, they were primarily characterized by increased DR in the presymptomatic AD group. Regions showing decreased FA and increased DR were the cingulum, a portion of the ILF, and portions of the IFOF. The pattern of decreased FA along with increased DR has been linked with loss of myelin in multiple sclerosis (Ciccarelli et al., 2006) and in animal studies of experimentally induced myelin loss (Song et al., 2002, 2005; Sun et al., 2006). The present pattern of DTIbased findings is would appear to be consistent with data demonstrating that myelin and its components such as cholesterol and myelin proteins are reduced early in the AD process (Han et al., 2002; Roher et al., 2002) and with a growing body of data suggesting that disruption of myelin integrity may be among the earliest events in the process of AD (Bartzokis, 2009). Based on this evidence, Bartzokis has proposed a model which reconceptualizes $\mathrm{AD}$ as initially a disease of demyelination, with classic amyloid beta and tau pathologies seen as by-products of the resulting homeostatic repair processes. The present findings of increased DR in the absence of changes in MD or MTL structural atrophy would appear to be consistent with DTI-based predictions that could be generated from the Bartzokis (2009) myelin hypothesis of AD.

The present study is the first to explore the DTI metrics FA/MD/DA/DR across the entire brain in presymptomatic AD. It is thus relevant to compare our findings with the few studies that have explored these DTI metrics across the brain in symptomatic AD (AcostaCabronero et al., 2010; Bosch et al., in press; Salat et al., 2010; Stricker et al., 2009). In contrast to the present results in presymptomatic $\mathrm{AD}$, each of the symptomatic $\mathrm{AD}$ studies 
except that of Stricker et al. (2009) reported that WM integrity reductions included increases in DA (in addition to DR increases). Of particular relevance was the study by Bosch et al. (in press), which included an MCI group in addition to $\mathrm{AD}$ and normal senior groups. These authors reported that, whereas both patient groups showed DR increases compared to a normal group, only the $\mathrm{AD}$ group showed $\mathrm{DA}$ increases. Taken together, the present findings and those from AD studies are consistent with suggestions that increased DA may occur at a relatively late stage of WM damage, possibly when 'cellular debris' is cleared by microglia (Concha et al., 2006; @ @ Sun et al., 2008). Of relevance, in contrast to the present results, the prior studies reporting increased DA also reported decreased MTL volume in symptomatic AD (Acosta-Cabronero et al., 2010; Bosch et al., in press; Salat et al., 2010). An important topic for future research will be to determine the timing of significant DA changes and their relationship to MTL volume during the course of AD.

At first pass, our findings would appear to be inconsistent with the possibility that Wallerian degeneration represents the primary mechanism of decreased WM integrity preceding AD. Wallerian degeneration secondary to distal cortical atrophy would be most clearly supported by findings of reduced volume of MTL regions, increased MD and altered DA. This pattern of results was not observed in our study. Instead, in the present study, the high AD-risk group showed no evidence of MTL degeneration and minimal evidence of gross tissue loss associated with increased MD. Similarly, the high AD-risk group showed only a few scattered voxels in which DA was altered compared to the low risk group. However, it is important to note that any specific conclusion about neurobiological mechanism underlying DTI-based WM declines remain speculative at this point. The DTI technique does not yet provide an unequivocal link to specific neurobiological mechanisms. Thus, it is not yet possible to definitively identify mechanisms such as 'demyelination,' or 'axonal degeneration' from DTI data (see Wheeler-Kingshott and Cercignani, 2009 and Hui et al., 2010 for relevant discussions).

Another reason for caution with respect to conclusions of demyelination or axonal degeneration from DTI data is that reduced WM integrity may in part reflect neuronal dysfunction. For example, we believe that the pattern of WM alterations observed in our study could be due to faulty communication between neuron and oligodendrocyte. Faulty communication could be mediated from the axonal side due to neuronal dysfunction, even in the absence of gross cortical atrophy. This possibility takes into account the concept that the axon and enveloping central myelin make up a unit integrated via intracellular signaling. The signaling scheme between axons and investing glia, particularly oligodendroglia in the CNS, is not yet well defined (Nave and Trapp, 2008). However, in the peripheral nervous system axonal signals are required for myelin maintenance, including $\operatorname{Pr}^{\mathrm{c}}$ a putative receptor for beta-amyloid (Bremer et al., 2010). Alterations in DR from myelin disruption in tracts projecting from pathologically involved areas could be reporting neuronal dysfunction through this mechanism at a very early stage, before volume changes are detectable in grey matter.

The present study has several limitations. First, as with other presymptomatic studies, a future clinical diagnosis of $\mathrm{AD}$ cannot yet be confirmed. The first step in the validation of the current findings will be to follow these currently normal study subjects longitudinally to determine which of the observed WM microstructural alterations are most predictive of later clinically diagnosed MCI or $\mathrm{AD}$. The conversion to MCI/AD is likely to be associated with multiple pathophysiologic mechanisms. Macrostructural degeneration of MTL structures is clearly among the important mechanisms. In addition, structural WM disconnection has been shown to contribute to age-related cognitive declines (Madden et al., 2004, 2009; Gold et al., 2010) and thus may also contribute to cognitive decline associated with the onset of $\mathrm{MCI} / \mathrm{AD}$. In particular, the continued loss of anatomical connectivity between MTL 
structures and posterior cingulate-retrosplenial cortices (i.e., further WM integrity reductions in the caudal cingulum tract) may be of special relevance given the importance of MTL and retrosplenial regions in memory processes (Vann et al., 2009).

Another caveat common to cross-sectional studies is that results could have been influenced by factors other than the variable of interest alone (in this case AD-risk). Although groups were carefully matched on a wide range of demographic, neuropsychological and medical health characteristics, it is possible that they differed in some other unknown way for which we could not adjust or control. Finally, we are not yet able to definitively link the observed WM alterations with specific underlying pathologies. Although different DTI-based diffusivity patterns have been linked with unique underlying pathologies in previous studies, we have no independent verification of pathology (e.g., by autopsy or brain biopsy) in our participants.

In conclusion, our results demonstrate DTI-based reductions in anatomical connectivity between neocortical and MTL allocortical regions in presymptomatic AD in the absence of detectable macrostructural MTL degeneration. The primary pattern of DTI results suggests that degeneration of WM tracts in presymptomatic $\mathrm{AD}$ appears to be associated with subtle decreases in axonal/myelin integrity. In a smaller number of tracts, WM reductions were characterized by decreased FA and increased DR, raising the possibility that a myelinrelated pathology could precede frank AD in some WM pathways.

\section{Acknowledgments}

This study was supported by NINDS Grant R01 NS-36660, NIA Grant R01 AG033036 and NSF Grant BCS-0814302.We thank Agnes Bognar, R.T., Kim Wilson, B.A., LuAnn Hamon, B.A., Dorothy Ross, B.A., and Barbara Martin, B.A. for their assistance in recruiting, scanning and testing the participants. We also thank two anonymous reviewers for their helpful comments.

\section{Glossary}

Abbreviations

MMSE

LM Imm

LM Delay

VR Imm

VR Delay

Block Des

BNT

SRT Imm

SRT Delay

Trails B - A

SS

Vocab

DS

LF
Mini Mental State Examination

Logical Memory Immediate

Logical Memory Delayed

Visual Reproduction Immediate

Visual Reproduction Delayed

Block Design

15 item Boston Naming Test

Selective Reminding Test Immediate

Selective Reminding Test Delayed

score on Trials B minus score on Trails A (to assess executive functioning after controlling for motor speed)

Spatial Span

Vocabulary

Digit Span

FAS Letter Fluency 


\section{References}

Acosta-Cabronero J, Williams GB, Pengas G, Nestor PJ. Absolute diffusivities define the landscape of white matter degeneration in Alzheimer's disease. Brain 2010;133:529-539. [PubMed: 19914928]

Assaf Y, Pasternak O. Diffusion tensor imaging (DTI)-based white matter mapping in brain research: a review. J Mol Neurosci 2008;34:51-61. [PubMed: 18157658]

Bartzokis G. Alzheimer's disease as homeostatic responses to age-related myelin breakdown. Neurobiol Aging. 2009

Basser PJ, Mattiello J, LeBihan D. Estimation of the effective self-diffusion tensor from the NMR spin echo. J Magn Reson B 1994;103:247-254. [PubMed: 8019776]

Basser PJ, Pierpaoli C. Microstructural and physiological features of tissues elucidated by quantitativediffusion-tensor MRI. J Magn Reson B 1996;111:209-219. [PubMed: 8661285]

Bennett IJ, Madden DJ, Vaidya CJ, Howard DV, Howard JH Jr. Age-related differences in multiple measures of white-matter integrity: A diffusion tensor imaging study of healthy aging. Hum Brain Mapp 2010;31:378-390. [PubMed: 19662658]

Bosch B, Arenaza-Urquijo EM, Rami L, Sala-Llonch R, Junque C, Sole-Padulles C, et al. Multiple DTI index analysis in normal aging, amnestic MCI and AD. Relationship with neuropsychological performance. Neurobiol Aging. (in press).

Braak H, Braak E. Neuropathological stageing of Alzheimer-related changes. Acta Neuropathol 1991;82:239-259. [PubMed: 1759558]

Braak H, Braak E. Development of Alzheimer-related neurofibrillary changes in the neocortex inversely recapitulates cortical myelogenesis. Acta Neuropathol 1996;92:197-201. [PubMed: 8841666]

Bremer J, Baumann F, Tiberi C, Wessig C, Fischer H, Schwarz P, Steele AD, Toyka KV, Nave KA, Weis J, Aguzzi A. Axonal prion protein is required for peripheral myelin maintenance. Nat Neurosci 2010;13:310-318. [PubMed: 20098419]

Brun A, Englund E. A white matter disorder in dementia of the Alzheimer type: a pathoanatomical study. Ann Neurol 1986;19:253-262. [PubMed: 3963770]

Burzynska AZ, Preuschhof C, Backman L, Nyberg L, Li SC, Lindenberger U, Heekeren HR. Agerelated differences in white matter microstructure: Region-specific patterns of diffusivity. Neuroimage. 2009

Catani M, Howard RJ, Pajevic S, Jones DK. Virtual in vivo interactive dissection of white matter fasciculi in the human brain. Neuroimage 2002;17:77-94. [PubMed: 12482069]

Ciccarelli O, Behrens TE, Altmann DR, Orrell RW, Howard RS, Johansen-Berg H, Miller DH, Matthews PM, Thompson AJ. Probabilistic diffusion tractography: a potential tool to assess the rate of disease progression in amyotrophic lateral sclerosis. Brain 2006;129:1859-1871. [PubMed: 16672290]

Colchester A, Kingsley D, Lasserson D, Kendall B, Bello F, Rush C, Stevens TG, Goodman G, Heilpern G, Stanhope N, Kopelman MD. Structural MRI volumetric analysis in patients with organic amnesia, 1: methods and comparative findings across diagnostic groups. J Neurol Neurosurg Psychiatry 2001;71:13-22. [PubMed: 11413256]

Concha L, Gross DW, Wheatley BM, Beaulieu C. Diffusion tensor imaging of time-dependent axonal and myelin degradation after corpus callosotomy in epilepsy patients. Neuroimage 2006;32:1090 1099. [PubMed: 16765064]

Convit A, De Leon MJ, Tarshish C, De Santi S, Tsui W, Rusinek H, George A. Specific hippocampal volume reductions in individuals at risk for Alzheimer's disease. Neurobiol Aging 1997;18:131138. [PubMed: 9258889]

de Leon MJ, DeSanti S, Zinkowski R, Mehta PD, Pratico D, Segal S, Clark C, Kerkman D, DeBernardis J, Li J, Lair L, Reisberg B, Tsui W, Rusinek H. MRI and CSF studies in the early diagnosis of Alzheimer's disease. J Intern Med 2004;256:205-223. [PubMed: 15324364]

Desikan RS, Segonne F, Fischl B, Quinn BT, Dickerson BC, Blacker D, Buckner RL, Dale AM, Maguire RP, Hyman BT, Albert MS, Killiany RJ. An automated labeling system for subdividing the human cerebral cortex on MRI scans into gyral based regions of interest. Neuroimage 2006;31:968-980. [PubMed: 16530430] 
Englund E, Brun A, Alling C. White matter changes in dementia of Alzheimer's type. Biochemical and neuropathological correlates. Brain 1988;111(Pt 6):1425-1439. [PubMed: 3208064]

Fellgiebel A, Wille P, Muller MJ, Winterer G, Scheurich A, Vucurevic G, Schmidt LG, Stoeter P. Ultrastructural hippocampal and white matter alterations in mild cognitive impairment: a diffusion tensor imaging study. Dement Geriatr Cogn Disord 2004;18:101-108. [PubMed: 15087585]

Fischl B, Salat DH, Busa E, Albert M, Dieterich M, Haselgrove C, van der Kouwe A, Killiany R, Kennedy D, Klaveness S, Montillo A, Makris N, Rosen B, Dale AM. Whole brain segmentation: automated labeling of neuroanatomical structures in the human brain. Neuron 2002;33:341-355. [PubMed: 11832223]

Fischl B, Salat DH, van der Kouwe AJ, Makris N, Segonne F, Quinn BT, Dale AM. Sequenceindependent segmentation of magnetic resonance images. Neuroimage 2004a;23 Suppl 1:S69S84. [PubMed: 15501102]

Fischl B, van der Kouwe A, Destrieux C, Halgren E, Segonne F, Salat DH, Busa E, Seidman LJ, Goldstein J, Kennedy D, Caviness V, Makris N, Rosen B, Dale AM. Automatically parcellating the human cerebral cortex. Cereb Cortex 2004b;14:11-22. [PubMed: 14654453]

Gold BT, Powell DK, Xuan L, Jicha GA, Smith CD. Age-related slowing of task switching is associated with decreased integrity of frontoparietal white matter. Neurobiol Aging 2010;31:512522. [PubMed: 18495298]

Han X, D MH, McKeel DW Jr, Kelley J, Morris JC. Substantial sulfatide deficiency and ceramide elevation in very early Alzheimer's disease: potential role in disease pathogenesis. J Neurochem 2002;82:809-818. [PubMed: 12358786]

Hsu JL, Leemans A, Bai CH, Lee CH, Tsai YF, Chiu HC, Chen WH. Gender differences and agerelated white matter changes of the human brain: a diffusion tensor imaging study. Neuroimage 2008;39:566-577. [PubMed: 17951075]

Hui ES, Cheung MM, Chan KC, Wu EX. B-value dependence of DTI quantitation and sensitivity in detecting neural tissue changes. Neuroimage 2010;49:2366-2374. [PubMed: 19837181]

Hyman BT, Van Hoesen GW, Damasio AR, Barnes CL. Alzheimer's disease: cell-specific pathology isolates the hippocampal formation. Science 1984;225:1168-1170. [PubMed: 6474172]

Jack CR Jr, Petersen RC, Xu YC, Waring SC, O'Brien PC, Tangalos EG, Smith GE, Ivnik RJ, Kokmen E. Medial temporal atrophy on MRI in normal aging and very mild Alzheimer's disease. Neurology 1997;49:786-794. [PubMed: 9305341]

Jones DK, Symms MR, Cercignani M, Howard RJ. The effect of filter size on VBM analyses of DTMRI data. Neuroimage 2005;26:546-554. [PubMed: 15907311]

Madden DJ, Whiting WL, Huettel SA, White LE, MacFall JR, Provenzale JM. Diffusion tensor imaging of adult age differences in cerebral white matter: relation to response time. Neuroimage 2004;21:1174-1181. [PubMed: 15006684]

Madden DJ, Spaniol J, Costello MC, Bucur B, White LE, Cabeza R, Davis SW, Dennis NA, Provenzale JM, Huettel SA. Cerebral white matter integrity mediates adult age differences in cognitive performance. J Cogn Neurosci 2009;21:289-302. [PubMed: 18564054]

Martin SB, Smith CD, Collins HR, Schmitt FA, Gold BT. Evidence that volume of anterior medial temporal lobe is reduced in seniors destined for mild cognitive impairment. Neurobiol Aging. 2008 (doi:10.1016/j.neurobiolaging.2008.08.010).

Medina D, DeToledo-Morrell L, Urresta F, Gabrieli JD, Moseley M, Fleischman D, Bennett DA, Leurgans S, Turner DA, Stebbins GT. White matter changes in mild cognitive impairment and AD: A diffusion tensor imaging study. Neurobiol Aging 2006;27:663-672. [PubMed: 16005548]

Mufson EJ, Pandya DN. Some observations on the course and composition of the cingulum bundle in the rhesus monkey. J Comp Neurol 1984;225:31-43. [PubMed: 6725639]

Nave KA, Trapp BD. Axon-glial signaling and the glial support of axon function. Annu Rev Neurosci 2008;31:535-561. [PubMed: 18558866]

Nierenberg J, Pomara N, Hoptman MJ, Sidtis JJ, Ardekani BA, Lim KO. Abnormal white matter integrity in healthy apolipoprotein E epsilon4 carriers. Neuroreport 2005;16:1369-1372. [PubMed: 16056141] 
Persson J, Lind J, Larsson A, Ingvar M, Cruts M, Van Broeckhoven C, Adolfsson R, Nilsson LG, Nyberg L. Altered brain white matter integrity in healthy carriers of the APOE epsilon4 allele: a risk for AD? Neurology 2006;66:1029-1033. [PubMed: 16606914]

Pierpaoli C, Basser PJ. Toward a quantitative assessment of diffusion anisotropy. Magn Reson Med 1996;36:893-906. [PubMed: 8946355]

Roher AE, Weiss N, Kokjohn TA, Kuo YM, Kalback W, Anthony J, Watson D, Luehrs DC, Sue L, Walker D, Emmerling M, Goux W, Beach T. Increased A beta peptides and reduced cholesterol and myelin proteins characterize white matter degeneration in Alzheimer's disease. Biochemistry 2002;41:11080-11090. [PubMed: 12220172]

Rueckert D, Sonoda LI, Hayes C, Hill DL, Leach MO, Hawkes DJ. Nonrigid registration using freeform deformations: application to breast MR images. IEEE Trans Med Imaging 1999;18:712-721. [PubMed: 10534053]

Salat DH, Tuch DS, van der Kouwe AJ, Greve DN, Pappu V, Lee SY, Hevelone ND, Zaleta AK, Growdon JH, Corkin S, Fischl B, Rosas HD. White matter pathology isolates the hippocampal formation in Alzheimer's disease. Neurobiol Aging 2010;31:244-256. [PubMed: 18455835]

Sen PN, Basser PJ. A model for diffusion in white matter in the brain. Biophys J 2005;89:2927-2938. [PubMed: 16100258]

Smith CD, Chebrolu H, Andersen AH, Powell DA, Lovell MA, Xiong S, Gold BT. White matter diffusion alterations in normal women at risk of Alzheimer's disease. Neurobiol Aging. 2008 (doi: 10.1016/j.neurobiolaging.2008.08.006).

Smith CD, Chebrolu H, Wekstein DR, Schmitt FA, Jicha GA, Cooper G, Markesbery WR. Brain structural alterations before mild cognitive impairment. Neurology 2007;68:1268-1273. [PubMed: 17438217]

Smith SM, Jenkinson M, Johansen-Berg H, Rueckert D, Nichols TE, Mackay CE, Watkins KE, Ciccarelli O, Cader MZ, Matthews PM, Behrens TE. Tract-based spatial statistics: voxelwise analysis of multi-subject diffusion data. Neuroimage 2006;31:1487-1505. [PubMed: 16624579]

Song SK, Sun SW, Ramsbottom MJ, Chang C, Russell J, Cross AH. Dysmyelination revealed through MRI as increased radial (but unchanged axial) diffusion of water. Neuroimage 2002;17:14291436. [PubMed: 12414282]

Song SK, Yoshino J, Le TQ, Lin SJ, Sun SW, Cross AH, Armstrong RC. Demyelination increases radial diffusivity in corpus callosum of mouse brain. Neuroimage 2005;26:132-140. [PubMed: 15862213]

Stebbins GT, Murphy CM. Diffusion tensor imaging in Alzheimer's disease and mild cognitive impairment. Behav Neurol 2009;21:39-49. [PubMed: 19847044]

Stricker NH, Schweinsburg BC, Delano-Wood L, Wierenga CE, Bangen KJ, Haaland KY, Frank LR, Salmon DP, Bondi MW. Decreased white matter integrity in late-myelinating fiber pathways in Alzheimer's disease supports retrogenesis. Neuroimage 2009;45:10-16. [PubMed: 19100839]

Sun SW, Liang HF, Trinkaus K, Cross AH, Armstrong RC, Song SK. Noninvasive detection of cuprizone induced axonal damage and demyelination in the mouse corpus callosum. Magn Reson Med 2006;55:302-308. [PubMed: 16408263]

Vann SD, Aggleton JP, Maguire EA. What does the retrosplenial cortex do? Nat Rev Neurosci 2009;10:792-802. [PubMed: 19812579]

Wheeler-Kingshott CAM, Cercignani M. About "axial" and "radial” diffusivities. Mag Reson in med 2009;61:1255-1260.

Zhang Y, Schuff N, Jahng GH, Bayne W, Mori S, Schad L, Mueller S, Du AT, Kramer JH, Yaffe K, Chui H, Jagust WJ, Miller BL, Weiner MW. Diffusion tensor imaging of cingulum fibers in mild cognitive impairment and Alzheimer disease. Neurology 2007;68:13-19. [PubMed: 17200485] 

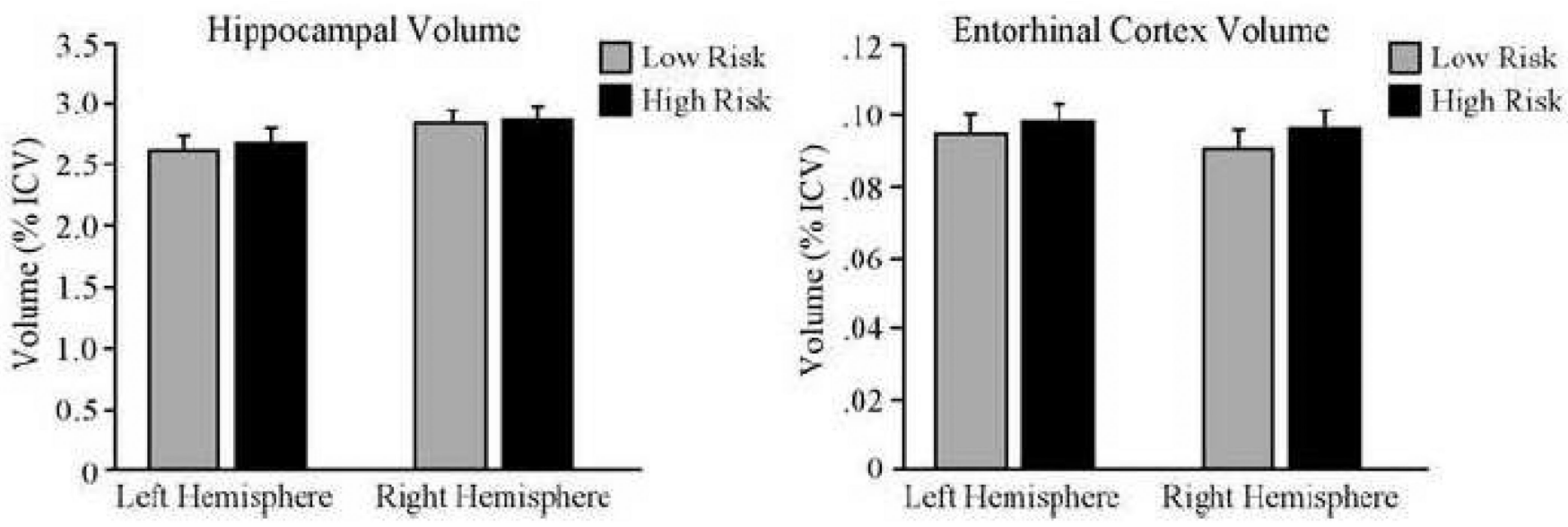

Figure 1.

Mean hippocampal and entorhinal cortex volumes in normal high and low AD-risk groups. Volumes are normalized as a percentage of intracranial volume (ICV) for visualization purposes. Bars represent the standard error of the mean. 


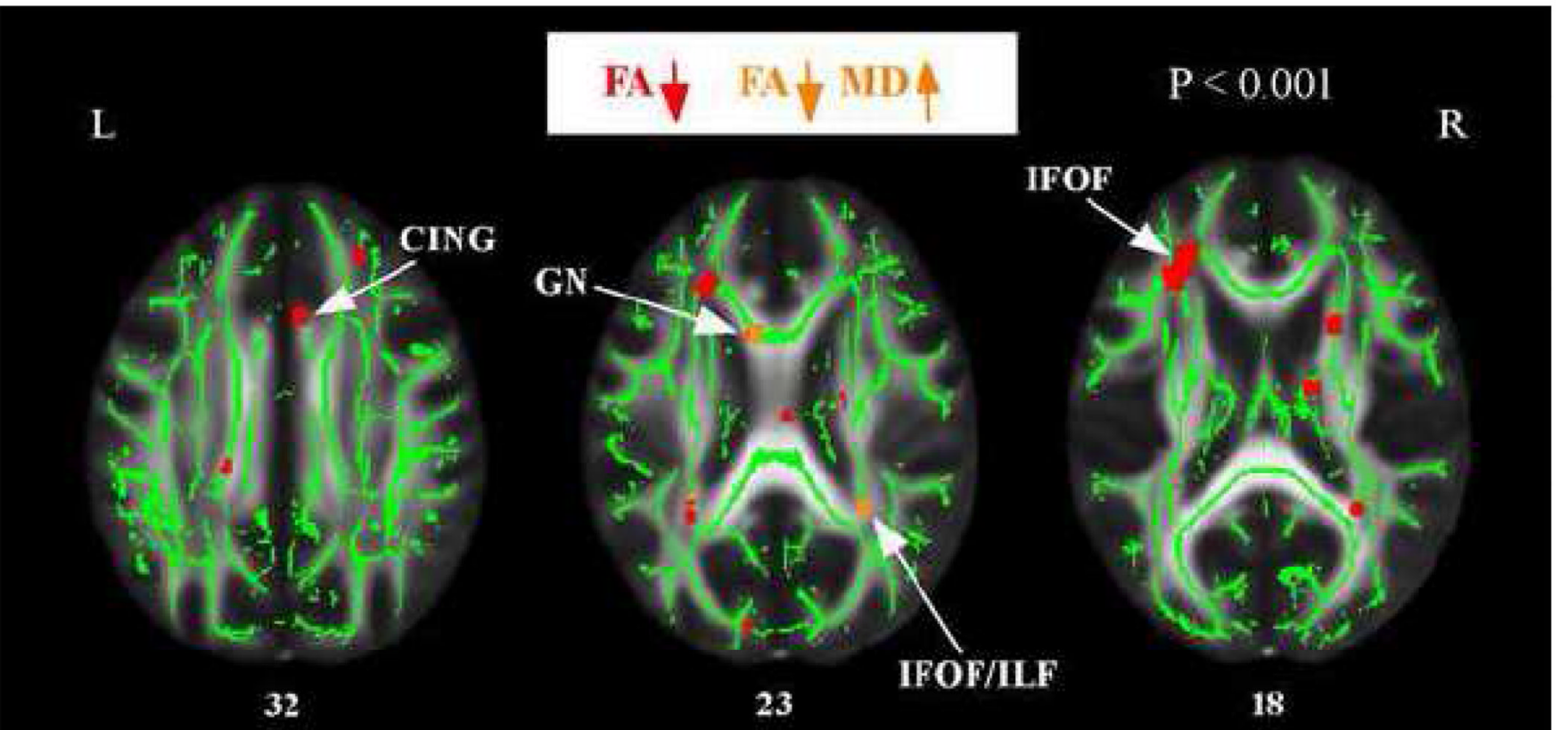

Figure 2.

Regions of decreased fractional anisotropy and increased mean diffusivity in normal, highAD risk subjects. The anatomic underlay used for illustration is the MNI-space registered target fractional anisotropy (FA) image. The registered average FA skeleton is represented in green. Regions of decreased FA are displayed in red. Regions of decreased FA and increased mean diffusivity (MD) are displayed in orange. The numbers in front of horizontal sections represent $\mathrm{z}$ coordinates in MNI space. 


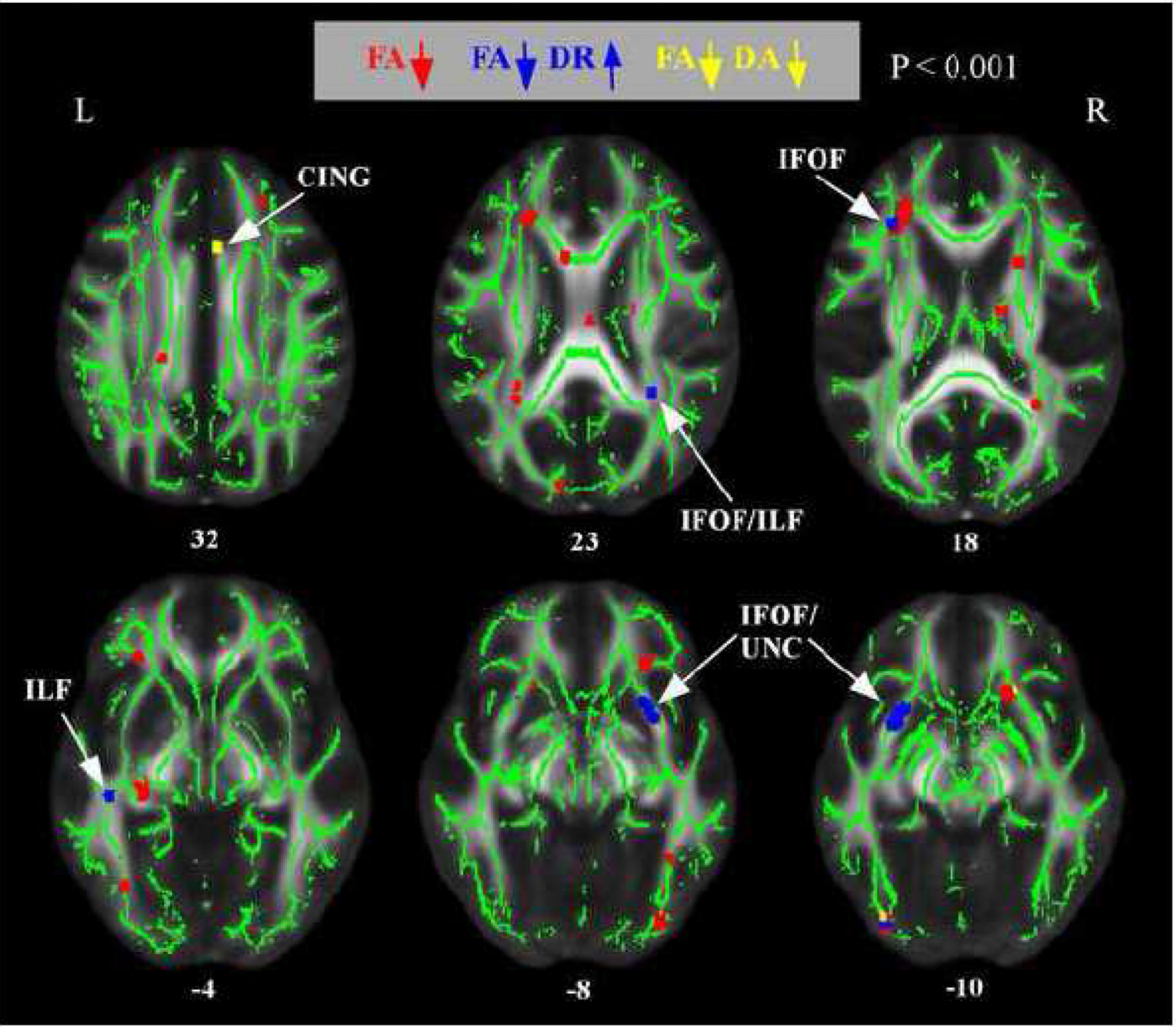

Figure 3.

Regions of decreased FA and altered radial or axial diffusivity in normal, high-AD risk subjects. The anatomic underlay and registered average FA skeleton are described in the Figure 1 legend. Regions of decreased FA are displayed in red. Regions of decreased FA and increased DR are displayed in dark blue. Regions of decreased FA and decreased DA are displayed in yellow. The numbers in front of horizontal sections represent $\mathrm{z}$ coordinates in MNI space. 


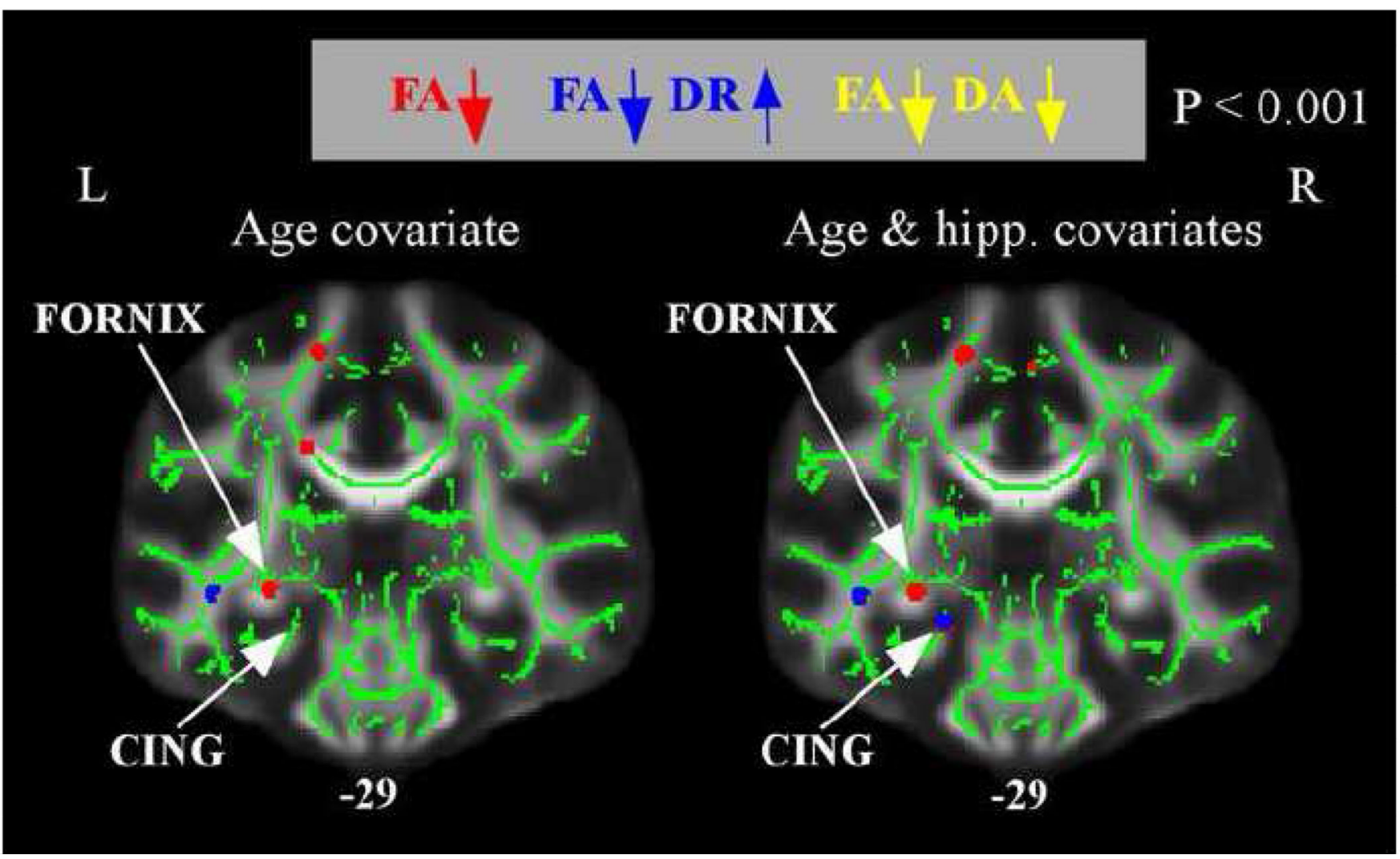

Figure 4.

Regions of decreased FA and altered radial or axial diffusivity in normal, high-AD risk subjects after controlling for hippocampal volume. The anatomic underlay and registered average FA skeleton are described in the Figure 1 legend. The left panel displays a coronal slice when only age was included as a covariate (as in results from Figures 2 and 3). The right panel displays the same coronal slice when normalized hippocampal volume, in addition to age, was included as a covariate. The numbers below the coronal sections represent y coordinates in MNI space. Controlling for hippocampal volume revealed an additional region of decreased FA and increased DR in the caudal portion of the cingulum. 


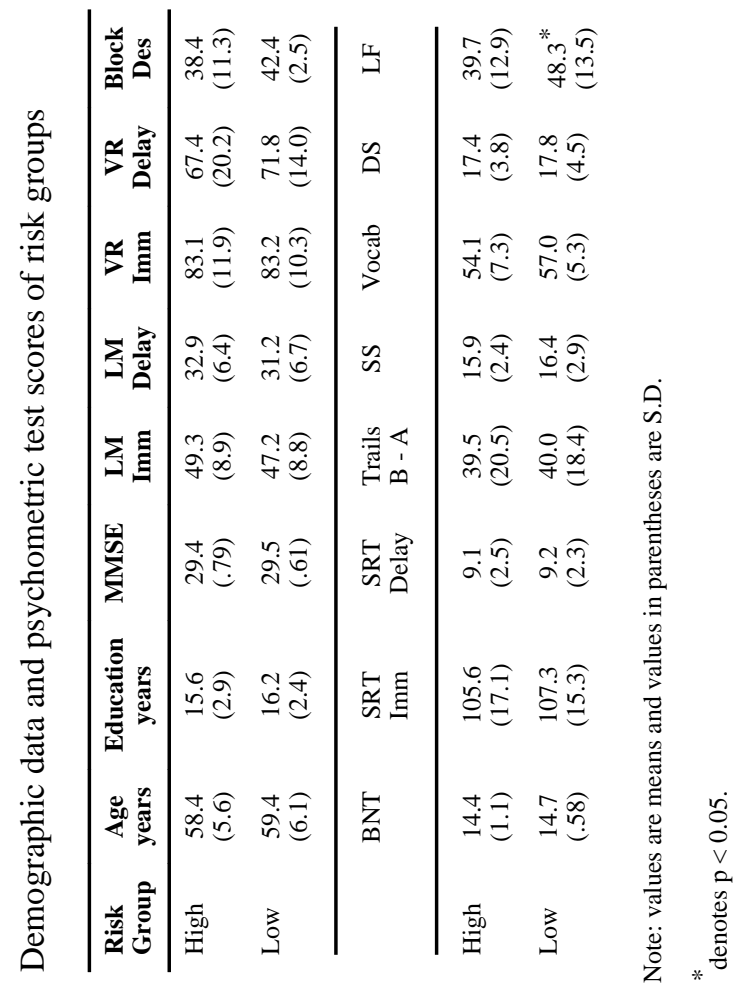

